\title{
Formulación de Preguntas en Medicina Basada en la Evidencia
}

\author{
Mauricio Soto ${ }^{1}$, G abriel Rada 2
}

F rente a diversos pacientes de nuestra práctica clínica, nos surgen dudas que generan necesidad de nueva información. Estas dudas pueden aparecer al momento del diagnóstico, al explorar posibles causas o diagnósticos diferenciales, al considerar un tratamiento o al dar un pronóstico.

En general, las dudas generan preguntas que podemos clasificar en dos tipos ${ }^{1}$ :

1. Preguntas básicas o de preparación (background): referentes al conocimiento general de una condición. Ej: ¿qué es la fibrosis quística?

2. Preguntas clínicas o de acción (foreground): sobre conocimientos específicos acerca del manejo de los pacientes con una determinada condición. Son, por tanto, interrogantes orientadas al paciente, aplicables en tiempo real»y con repercusión directa en la toma de decisiones.

El primer tipo de preguntas se responde usando como fuentes de información los textos generales (libros de referencia, artículos de revisión).

En el caso de las preguntas clínicas específicas, encontramos muchas veces que los textos 0 revisiones generales no son capaces de respondernos y debemos recurrir entonces a fuentes más específicas como los estudios publicados en revistas científicas. Lamentablemente, no es fácil encontrar lo que buscamos en el enorme volumen de publicaciones científicas ${ }^{2}$. Surge así la necesidad de una adecuada formulación de la pregunta que permita obtener una respuesta pertinente en forma eficiente.

\section{Tabla 1. Etapas en la práctica de la M edicina Basada en Evidencia}

\author{
1. Convertir el problema clínico en una pregunta. \\ 2. Buscar la «evidencia» en la literatura \\ 3. Análisis crítico de la evidencia \\ 4. Aplicar los resultados en la práctica clínica
}

Transformar las dudas en preguntas clínicas, constituye la primera etapa de la Medicina Basada en Evidencia (Tabla 1).

Diversos estudios han evaluado la cantidad de dudas que tienen los clínicos. En un estudio inglés se observó que en un día de práctica habitual, cada médico familiar se hizo 2 preguntas por cada 3 pacientes. El $40 \%$ de las preguntas fue de información puntual (Ej. ¿cuál es la dosis?). El estudio reveló que $64 \%$ de las preguntas no son respondidas inmediatamente, y que se generaban 4 preguntas sin respuestas por cada medio día de trabajo. Las preguntas respondidas en presencia del paciente ocupaban en promedio 2 minutos al clínico ${ }^{3}$.

\section{Tipos de preguntas clínicas}

Las preguntas clínicas pueden relacionarse con distintas áreas de la toma de decisiones, que requieren a su vez diferentes diseños de estudios para ser respondidas:

- Causalidad o etiología Cohorte, caso y control

- Pronóstico

Cohorte longitudinal

- Tamizaje o Screening

- Diagnóstico

- Terapia Estudio con Gold Standard Ensayo clínico randomizado

\footnotetext{
${ }^{1}$ Médico Familiar, Universidad Católica de Chile.

${ }^{2}$ Residente de Medicina Interna, Universidad Católica de Chile.

Revisado por Unidad de Medicina Basada en Evidencia UC (UMBE-UC)
} 
Tabla 2. Preguntas clínicas: D efiniendo palabras claves para una búsqueda eficiente

\begin{tabular}{|lccccc|}
\hline & Pacientes & Intervención & Comparación & Resultados & Área \\
\hline $\begin{array}{l}\text { Palabras } \\
\text { claves }\end{array}$ & Hipertensos & $\begin{array}{c}\text { Amlodipino } \\
\text { o lisinopril }\end{array}$ & Diuréticos & $\begin{array}{c}\text { Mortalidad } \\
\text { cardiovascular }\end{array}$ & Terapia \\
& $\begin{array}{c}\text { Sospecha de } \\
\text { TEP* }\end{array}$ & Dímero D & & TEP & Diagnóstico \\
\hline
\end{tabular}

*TEP: Tromboembolismo pulmonar

Componentes de la pregunta clínica

En una pregunta clínica bien estructurada pueden identificarse 3 ó 4 componentes principales:

- Paciente o población o problema de interés: ¿Podría describir un grupo de pacientes similar al que me interesa?

- Intervención a considerar: ¿Cuál es la principal intervención que quiero llevar a cabo?

- Comparación: ¿Qué otras opciones existen?

- Outcome o resultado: ¿Qué esperamos que le suceda al paciente?

Esta forma de estructurar la pregunta se aplica principalmente a preguntas de tratamiento. Por ejemplo: ¿En pacientes hipertensos, puede el tratamiento con amlodipino o lisinopril comparado con diuréticos tiazídicos reducir la morbimortalidad cardiovascular?

Al descomponer la pregunta en sus componentes tenemos: Pacientes: Hipertensos. Intervención: Amlodipino o lisinopril. Comparación: Diuréticos tiazídicos. Outcome: Mortalidad cardiovascular.

En el caso de otro tipo de preguntas se recomienda usar sólo tres componentes:

\section{REFERENCIAS}

1. GuyatT G. Users' Guides to the Medical Literature. American Medical Association. 2002.
Paciente, población o problema, Intervención (test o examen diagnóstico) y Outcome. Ej: ¿En pacientes con sospecha de tromboembolismo pulmonar, me permite el dímero $\mathrm{D}$ descartar la presencia de tromboembolismo? (Tabla 2).

Transformar las necesidades de información en una pregunta clínica bien estructurada y dividirla en sus componentes permite planificar la estrategia de búsqueda más eficiente, al facilitar el proceso de elección de términos claves a utilizar en el buscador (Tabla 2). Seleccionar palabras desde los dos primeros componentes de la pregunta permite encontrar artículos relevantes en la mayoría de los casos.

En suma, este primer paso surge en relación a un paciente. El clínico incorpora la necesidad de resolver la incertidumbre. La pregunta bien estructurada facilita la identificación de una estrategia de búsqueda para poder encontrar artículos relevantes que luego serán analizados críticamente, para finalmente volver al paciente, quien es en definitiva el beneficiario final de todo el proceso de la Medicina Basada en Evidencia. (Tabla 1).

2. ELY J. Obstacles to answering doctors' questions about patient care with evidence: qualitative study. BMJ 2002; 324: 710.

3. EIY J. Analysis of questions asked by family doctors regarding patient care. BMJ 1999; 319: 358-61. 